\title{
Stability Analysis for Grain Yield and Its Attributing Traits of Rice across Locations
}

\author{
Patel Unnati $^{*}$, Patel Pathik ${ }^{2}$, Mahatma Lalit ${ }^{3}$ and Bhimshinh Bilwal ${ }^{1}$ \\ ${ }^{1}$ Department of Genetics and Plant Breeding, Navsari Agricultural University, Gujarat, India \\ ${ }^{2}$ Main Rice Research Centre, Navsari Agricultural University, Gujarat, India \\ ${ }^{3}$ Department of plant pathology, Navsari Agricultural University, Gujarat, India \\ *Corresponding author
}

\section{A B S T R A C T}

Forty rice hybrids along with their parents were evaluated for their stability parameters with respect to yield and its attributing characters like Days to 50 per cent flowering, Productive tillers per plant, Plant height $(\mathrm{cm})$, Panicle length $(\mathrm{cm})$, Grains per panicle, Grain yield per plant $(\mathrm{g})$, Straw yield per plant $(\mathrm{g})$ and test weight $(\mathrm{g})$ in a multi-locational

\section{Keywords}

Grain yield, Stability, G x E interaction, Rice.

\section{Article Info}

Accepted:

17 September 2017

Available Online:

10 November 2017 trial at three different sites of Gujarat viz., Main Rice Research centre, Navsari, Regional Rice Research Station, Vyara and Hill Millet Research Station, Waghai during Kharif 2015. Pooled analysis of variance reflects existence of genotype x environment interaction. Stability analysis revealed significant differences among all the characters for genotype and environment except for test weight $(\mathrm{g})$ in environment. The results on environmental index revealed that Navsari was the most congenial location for grain yield per plant and majority of yield related traits in addition to productive tillers per plant, panicle length, grains per panicle and test weight. The best three high yielding and stable hybrids over environments for grain yield per plant were GAR-13 x IET-24767, NAUR-1 x IET-23833 and GAR-13 x IET-23833. The hybrid GAR-13 x IET-24767 was found to be stable for plant height $(\mathrm{cm})$, panicle length $(\mathrm{cm})$, grains per panicle and test weight $(\mathrm{g})$. Among the parents, male IET-24774 reflected better suitability for favourable environments for grain yield per plant.

\section{Introduction}

In India, rice is being grown as a major food crop under diverse agro-climatic conditions. Rice accounts for $35-60 \%$ of the caloric intake of three billion Asians (Guyer et al., 1998). It is very much necessary to develop varieties having stable yield performance over diverse environments. In breeding programme, it is necessary to screen and develop stable genotypes, which perform more or less uniform under varying environmental conditions. Thus knowledge of genotype $\mathrm{x}$ environment interaction helps the breeder to select high yielding and most adaptable varieties and hybrids. In any crop, plant breeders are mostly interested and continuously engaged in improvement of yield character. Yield is complex character which depends on many component characters, type of genotype, environmental conditions and genotype $\mathrm{x}$ environment interactions. Hence, knowledge on the nature and magnitude of genotype $\mathrm{x}$ environment 
interactions is important in understanding the stability of a particular variety before it is being recommended for a given situation. Testing of genotypes under different environments differing in unpredictable variation is an accepted approach for selecting stable genotypes (Eberhart and Russel, 1966). Present Investigation reports these facts with rice crop.

\section{Materials and Methods}

The present experiment material comprised of four lines, ten testers, their forty hybrids and two checks. The trials were conducted in a randomized block design with three replications at three locations viz., Main Rice Research centre, Navsari, Regional Rice Research Station, Vyara and Hill Millet Research Station, Waghai representing diverse agro climatic condition during Kharif 2015. Observations were recorded on five randomly selected plants in each replication in each environment in respect of eight quantitative characters viz., Days to fifty per cent flowering, Number of productive tillers per plant, Plant height $(\mathrm{cm})$, Panicle length (cm), Grains per panicle, Grain yield per plant (g), Straw yield per plant (g) and Test weight (g). The statistical analysis for genotype $\mathrm{x}$ environment interactions and stability parameters were worked out according to Eberhart and Russell (1966) for all the characters under study.

\section{Results and Discussion}

Pooled analysis of variance showed highly significant mean sum of squares for genotypes and environments for all the characters studied, indicating the presence of substantial variation among the genotypes over environments (Table 1). Mean sum of squares due to genotypes found highly significant for all the characters when tested against pooled error and pooled deviation.
The differences due to environment component were found highly significant except for test weight when tested against pooled error. The environment (linear) mean square was observed significant for all characters except for test weight when tested against pooled error. Coincidence of genotypic performance with environmental values for all the characters was observed due to significant Genotype $\mathrm{x}$ Environment (Linear) for all the traits except for plant height, indicated that cultivars performance may differ markedly for these characters. Significant pooled deviation found significant for all traits except for test weight (g) suggested that the performance of different genotypes fluctuated considerably in respect to their stability for respective characters. Thus both predictable and unpredictable components contributed significantly to differences in stability among genotypes. These results are in agreement to those reported by Nayak (2008), Shadakshari (2001) and Rashmi et al., (2017) in rice.

Environmental index can provide the basis for identifying the favourable environments for the expression of maximum potential of the genotype (Table 2). Navsari found to be congenial for days to 50 per cent flowering, numbers of productive tillers per plant, panicle length $(\mathrm{cm})$, grains per panicle, grain yield per plant $(\mathrm{g})$, straw yield per plant $(\mathrm{g})$ and test weight $(\mathrm{g})$. While it was favourable for days to 50 per cent flowering and plant height $(\mathrm{cm})$ at Vyara location and location Waghai was found to be favourable for plant height $(\mathrm{cm})$.

In the present, stability of 40 rice hybrid, 14 parental line and two check varieties with respect to 8 characters was judged by three parameters viz., mean (x), regression coefficient (bi) and deviation from regression $\left(S^{2} \mathrm{di}\right)$ using the model proposed by Eberhart and Russell (1966). 
Table.1 Pooled MSS values for phenotypic stability for different quantitative traits over three environments

\begin{tabular}{|c|c|c|c|c|c|c|c|c|c|}
\hline Source of variation & d.f. & $\begin{array}{l}\text { Days to } \\
50 \% \\
\text { flowering }\end{array}$ & $\begin{array}{l}\text { Productive } \\
\text { tillers/plant }\end{array}$ & $\begin{array}{l}\text { Plant height } \\
(\mathrm{cm})\end{array}$ & $\begin{array}{l}\text { Panicle } \\
\text { length }(\mathrm{cm})\end{array}$ & $\begin{array}{l}\text { Grains per } \\
\text { panicle }\end{array}$ & $\begin{array}{ll}\text { Grain } & \text { yield } \\
\text { per } & \text { plant } \\
\text { (g) } & \\
\end{array}$ & $\begin{array}{ll}\text { Straw } & \text { yield } \\
\text { per } & \text { plant } \\
\text { (g) } & \end{array}$ & $\begin{array}{l}\text { Test weight } \\
\text { (g) }\end{array}$ \\
\hline Genotypes (G) & 55 & $140.51^{* *++}$ & $18.29^{* *++}$ & $476.42^{* *++}$ & $32.64^{* *++}$ & $2753.31^{* *++}$ & $78.06^{* *++}$ & $112.61^{* *++}$ & $34.56^{* *++}$ \\
\hline Environments (E) & 2 & $40.23^{* *++}$ & $5.76^{*++}$ & $768.65^{* *++}$ & $4.82^{++}$ & $684.73^{* *++}$ & $94.28^{* *++}$ & $142.17^{* *++}$ & 0.62 \\
\hline $\mathrm{G} \times \mathrm{E}$ & 110 & $8.25^{++}$ & $1.55^{++}$ & 51.73 & $2.73^{++}$ & $91.03^{+}$ & $6.40^{++}$ & $8.49^{++}$ & 1.69 \\
\hline $\begin{array}{l}\text { Environments } \\
\text { (Linear) }\end{array}$ & 1 & $80.46^{* *++}$ & $11.52^{* *++}$ & $1537.30^{* *++}$ & $9.65^{++}$ & $1369.46^{* *++}$ & $188.55^{* *++}$ & $284.34^{* *++}$ & 1.24 \\
\hline Gx E (Linear) & 55 & $8.44^{++}$ & $1.82^{++}$ & 33.37 & $2.82^{++}$ & $100.62^{++}$ & $6.82^{++}$ & $8.65^{++}$ & $1.74^{+}$ \\
\hline Pooled deviation & 56 & $7.91 \mathrm{SS}$ & $1.26 \mathrm{SS}$ & $68.83 \mathrm{SS}$ & $2.60 \mathrm{SS}$ & $79.97 \mathrm{SS}$ & $5.87 \mathrm{SS}$ & $8.18 \mathrm{SS}$ & 1.62 \\
\hline Pooled error & 330 & 1.35 & 0.31 & 44.95 & 1.10 & 48.10 & 3.04 & 3.04 & 1.27 \\
\hline
\end{tabular}

SS = Significant when pooled deviation tested against pooled error,

$*$, ** - indicates $5 \%$ and $1 \%$ probability levels of significance, respectively tested against pooled deviation

,+++- indicates $5 \%$ and $1 \%$ probability levels of significance, respectively tested against pooled error.

Table.2 Estimates of environmental index for various traits under different environments in rice

\begin{tabular}{llll}
\hline \multirow{2}{*}{ Characters } & \multicolumn{2}{l}{ Environmental index } \\
\cline { 2 - 4 } & L-I & L-II & L-III \\
\hline Days to 50 per cent flowering & -0.35 & -0.62 & 0.97 \\
Productive tiles per plant & 0.34 & -0.05 & -0.29 \\
Plant height (cm) & 3.87 & -3.52 & -0.35 \\
Panicle length (cm) & 0.34 & -0.13 & -0.21 \\
Grains per panicle & 3.87 & -0.94 & -2.93 \\
Grain yield per plant(g) & 1.32 & -0.04 & -1.28 \\
Straw yield per plant (g) & 1.64 & -0.10 & -1.54 \\
Test weight (g) & 0.12 & -0.08 & -0.04 \\
\hline
\end{tabular}

Note: L-I: Navsari, L-II: Vyara, L-III: Waghai 
Table.3 Mean performance and stability parameters of different genotypes for different characters in rice

\begin{tabular}{|c|c|c|c|c|c|c|c|c|c|c|c|c|c|}
\hline \multirow{2}{*}{$\begin{array}{l}\text { Sr. } \\
\text { No }\end{array}$} & \multirow[t]{2}{*}{ Genotypes } & \multicolumn{3}{|c|}{ Days to $50 \%$ flowering } & \multicolumn{3}{|c|}{ Productive tillers per plant } & \multicolumn{3}{|c|}{ Plant height $(\mathrm{cm})$} & \multicolumn{3}{|c|}{ Panicle length $(\mathrm{cm})$} \\
\hline & & Mean & bi & $S^{2} \mathrm{di}$ & Mean & bi & $\mathbf{S}^{2} \mathrm{di}$ & Mean & bi & $\mathbf{S}^{2} \mathbf{d i}$ & Mean & bi & $S^{2} \mathbf{d i}$ \\
\hline 1. & NAUR-1 & 96.78 & 2.36 & $21.20 * *$ & 8.82 & 2.82 & 0.15 & 98.86 & 2.67 & -35.60 & 28.71 & 1.53 & -0.81 \\
\hline 2. & GNR-3 & 94.67 & 0.67 & -1.33 & 8.16 & $4.74^{*}$ & -0.30 & 127.54 & 0.39 & -11.72 & 24.73 & 2.92 & -0.98 \\
\hline 3. & Gurjari & 95.22 & 1.79 & $7.46^{*}$ & 7.07 & 1.36 & 0.84 & 127.74 & -1.06 & 47.45 & 18.89 & 2.41 & 3.20 \\
\hline 4. & GAR-13 & 101.89 & 1.25 & $9.81 * *$ & 7.53 & 1.81 & -0.23 & 126.44 & 1.59 & 19.07 & 22.94 & -0.49 & $9.59 * *$ \\
\hline 5. & NVSR-306-6 & 92.00 & -3.55 & -0.78 & 7.27 & -1.12 & 0.35 & 113.14 & -1.59 & $219.46^{*}$ & 18.72 & 6.87 & -1.09 \\
\hline 6. & NVSR-306-14 & 90.56 & -0.42 & $14.48 * *$ & 7.62 & 2.00 & -0.19 & 108.06 & 2.21 & 83.17 & 20.98 & -7.87 & 2.66 \\
\hline 7. & IET-23814 & 89.33 & 2.81 & 3.53 & 7.69 & $-0.63 *$ & -0.31 & 154.65 & 1.63 & $161.52 *$ & 16.72 & -1.39 & 2.08 \\
\hline 9. & IET-23829 & 98.78 & 2.12 & 0.50 & 8.27 & -2.32 & $0.89 *$ & 154.36 & 1.06 & 51.78 & 16.58 & -0.42 & -0.63 \\
\hline 10. & IET-24767 & 85.89 & 0.14 & $4.48 *$ & 10.20 & -0.94 & 0.16 & 131.10 & 3.03 & -42.18 & 25.04 & 1.24 & $8.38 * *$ \\
\hline 11. & IET-24774 & 94.00 & -0.43 & $9.27 * *$ & 9.29 & -0.70 & 0.04 & 127.00 & 1.54 & 33.89 & 20.94 & 7.70 & 0.07 \\
\hline 12. & IET-23832 & 96.00 & $-4.10 *$ & -1.25 & 9.27 & -2.80 & $2.39 * *$ & 121.99 & 2.15 & -18.32 & 18.65 & 6.62 & 0.69 \\
\hline 13. & IET-23833 & 81.11 & 2.49 & 0.70 & 10.73 & -0.68 & -0.02 & 108.10 & 1.16 & -1.78 & 26.31 & -3.60 & -0.92 \\
\hline 14. & IET-23834 & 82.44 & -0.88 & $8.72 * *$ & 9.42 & $-3.36^{*}$ & -0.31 & 145.38 & 0.80 & -0.52 & 18.12 & 3.70 & -0.08 \\
\hline & Parental mean & 92.62 & & & 8.49 & & & 126.31 & & & 20.99 & & \\
\hline 15. & NAUR-1 x NVSR-303-6 & 90.89 & -3.10 & -0.64 & 9.58 & 3.95 & $1.37 *$ & 114.13 & 1.31 & 44.26 & 22.92 & 2.51 & 0.63 \\
\hline 17. & NAUR-1 x IET-23814 & 85.33 & 6.98 & -1.03 & 10.24 & 1.98 & $1.84 * *$ & 139.36 & 0.74 & -10.43 & 23.95 & 0.18 & 0.17 \\
\hline 18. & NAUR-1 x IET-23825 & 92.67 & 3.19 & $8.94 * *$ & 10.21 & 4.12 & 0.58 & 129.46 & -0.81 & 52.38 & 23.61 & 4.23 & -1.03 \\
\hline 19. & NAUR-1 x IET-23829 & 94.89 & -0.23 & $5.32^{*}$ & 10.93 & 3.98 & 0.24 & 132.97 & 0.72 & -18.57 & 24.75 & 1.64 & -0.93 \\
\hline 20. & NAUR-1 x IET-24767 & 84.22 & 3.56 & $5.37 *$ & 15.04 & -3.35 & $1.62 *$ & 133.30 & 0.90 & -3.87 & 27.74 & 0.97 & -0.74 \\
\hline 21. & NAUR-1 x IET- 24774 & 86.00 & -3.67 & 1.49 & 11.96 & 0.34 & $1.91 * *$ & 130.04 & -0.49 & 2.60 & 26.19 & 0.15 & 1.95 \\
\hline 22. & NAUR-1 x IET-23832 & 90.33 & -1.95 & $10.74 *$ & 10.47 & -4.59 & 0.30 & 125.07 & 0.58 & -10.89 & 25.79 & -8.43 & 1.84 \\
\hline 23. & NAUR-1 x IET-23833 & 77.56 & -2.86 & 1.21 & 15.96 & 1.28 & 0.09 & 127.07 & -1.02 & 24.23 & 28.13 & 0.83 & 0.05 \\
\hline 24. & NAUR-1 x IET-23834 & 78.11 & 2.18 & -0.55 & 10.02 & 7.58 & 0.33 & 129.33 & 1.09 & 38.30 & 25.11 & 6.37 & -0.15 \\
\hline 25. & GNR-3 x NVSR-303-6 & 89.33 & 0.86 & $13.80 * *$ & 9.04 & -3.73 & $2.29 * *$ & 124.15 & 0.52 & 5.11 & 22.60 & -3.28 & -0.86 \\
\hline 26. & GNR-3 x NVSR-303-14 & 86.89 & 3.76 & 0.69 & 11.53 & -2.48 & $3.24 * *$ & 120.80 & -0.65 & 11.35 & 20.17 & -0.57 & -0.62 \\
\hline 27. & GNR-3 x IET-23814 & 87.67 & -0.25 & $18.78 * *$ & 8.91 & 3.91 & 0.05 & 122.07 & 0.88 & -0.38 & 21.12 & 1.57 & 0.81 \\
\hline 28. & GNR-3 x IET-23825 & 95.78 & 3.10 & -0.65 & 9.49 & 0.83 & $5.92 * *$ & 119.69 & 2.42 & -41.19 & 23.90 & 3.17 & $8.65 * *$ \\
\hline
\end{tabular}




\begin{tabular}{|c|c|c|c|c|c|c|c|c|c|c|c|c|c|}
\hline \multirow{2}{*}{$\begin{array}{l}\text { Sr. } \\
\text { No }\end{array}$} & \multirow[t]{2}{*}{ Genotypes } & \multicolumn{3}{|c|}{ Days to $50 \%$ flowering } & \multicolumn{3}{|c|}{ Productive tillers per plant } & \multicolumn{3}{|c|}{ Plant height (cm) } & \multicolumn{3}{|c|}{ Panicle length $(\mathrm{cm})$} \\
\hline & & Mean & bi & $\mathbf{S}^{2} \mathrm{di}$ & Mean & bi & $\mathrm{S}^{2} \mathrm{di}$ & Mean & bi & $\mathbf{S}^{2} \mathrm{di}$ & Mean & bi & $\mathbf{S}^{2} \mathrm{di}$ \\
\hline 29. & GNR-3 x IET-23829 & 96.56 & 0.44 & 6.00 & 9.64 & 2.26 & $5.01 * *$ & 117.70 & 0.73 & -11.66 & 23.35 & 2.27 & -0.25 \\
\hline 30. & GNR-3 x IET-24767 & 87.11 & 1.01 & $18.81^{* *}$ & 15.31 & 2.07 & $2.44 * *$ & 125.71 & 0.71 & 15.12 & 27.08 & -1.28 & 2.38 \\
\hline 31. & GNR-3 x IET-24774 & 88.11 & 3.04 & -1.21 & 12.31 & 4.82 & 0.23 & 124.26 & -0.81 & 7.11 & 21.69 & 7.08 & -0.84 \\
\hline 32. & GNR-3 x IET-23832 & 92.44 & 1.39 & $11.27 * *$ & 10.49 & $5.28 *$ & -0.29 & 145.50 & 1.16 & 16.22 & 22.65 & 5.19 & 2.01 \\
\hline 33. & GNR-3 x IET-23833 & 78.00 & 2.70 & 3.10 & 15.38 & 3.79 & -0.15 & 123.55 & 1.34 & 56.24 & 28.02 & -0.71 & -1.12 \\
\hline 34. & GNR-3 x IET-23834 & 79.67 & 1.10 & 3.15 & 9.07 & $-4.01 *$ & -0.30 & 126.71 & -0.66 & 16.42 & 21.15 & -1.37 & -0.97 \\
\hline 35. & Gurjari x NVSR-303-6 & 93.33 & 3.73 & -1.12 & 7.20 & -0.86 & 0.29 & 119.64 & 0.84 & -26.75 & 17.56 & -3.83 & 0.97 \\
\hline 37. & Gurjari x IET-23814 & 83.56 & -2.43 & -0.41 & 8.07 & -1.29 & $2.40 * *$ & 128.01 & 1.37 & 57.60 & 18.81 & 3.13 & 0.12 \\
\hline 38. & Gurjari x IET-23825 & 97.67 & 3.05 & $7.49 *$ & 8.29 & 0.33 & -0.13 & 116.17 & 2.28 & -40.88 & 18.42 & -2.33 & 0.33 \\
\hline 39. & Gurjari x IET-23829 & 96.11 & 3.84 & 0.03 & 7.36 & -0.74 & $1.63 *$ & 121.98 & 0.62 & 103.15 & 19.37 & 1.06 & 2.13 \\
\hline 40. & Gurjari x IET-24767 & 82.44 & -2.53 & $5.50^{*}$ & 14.42 & 4.83 & 0.16 & 121.69 & 1.04 & 54.72 & 24.67 & 4.69 & -0.99 \\
\hline 41. & Gurjari x IET-24774 & 86.89 & 1.30 & 0.73 & 10.40 & 4.10 & 0.13 & 106.58 & 1.80 & 99.30 & 20.69 & 6.34 & -0.57 \\
\hline 42. & Gurjari x IET-23832 & 90.11 & -1.18 & $4.29^{*}$ & 7.73 & -2.68 & -0.28 & 131.39 & -0.64 & 100.86 & 19.79 & -5.30 & -0.03 \\
\hline 43. & Gurjari x IET-23833 & 76.11 & 0.60 & $6.21 *$ & 14.84 & 4.04 & -0.03 & 131.53 & -0.72 & -8.40 & 24.54 & 6.00 & $3.43 *$ \\
\hline 44. & Gurjari x IET-23834 & 77.00 & $2.75 *$ & -1.35 & 7.91 & 3.47 & -0.20 & 121.27 & 2.01 & 114.08 & 20.54 & 5.25 & -0.76 \\
\hline 47. & GAR-13 x IET-23814 & 82.33 & -0.29 & $29.42 * *$ & 8.13 & -2.83 & $1.63^{*}$ & 128.34 & 2.20 & -34.52 & 20.48 & -3.65 & 1.69 \\
\hline 48. & GAR-13 x IET-23825 & 96.89 & 3.39 & $13.74 * *$ & 8.42 & 2.21 & $2.89 * *$ & 122.43 & 1.17 & 39.91 & 19.92 & 6.69 & 0.39 \\
\hline 49. & GAR-13 x IET-23829 & 97.22 & 2.66 & $11.24 * *$ & 6.76 & -1.95 & -0.29 & 138.96 & 1.16 & 32.29 & 21.18 & 0.14 & $14.75 * *$ \\
\hline 50. & GAR-13 x IET-24767 & 82.78 & 1.15 & $17.27 * *$ & 14.96 & 2.34 & $3.16^{* *}$ & 105.10 & 2.05 & -41.43 & 25.17 & 2.75 & -0.16 \\
\hline 51. & GAR-13 x IET-24774 & 90.11 & 4.02 & $5.26 *$ & 11.18 & -2.95 & -0.21 & 113.23 & 1.22 & 39.61 & 19.78 & -8.38 & 1.38 \\
\hline 52. & GAR-13 x IET-23832 & 99.56 & 0.27 & $12.84 * *$ & 6.64 & 1.33 & -0.29 & 107.78 & 2.25 & -6.70 & 20.25 & 6.06 & 0.55 \\
\hline 53. & GAR-13 x IET-23833 & 80.78 & 3.23 & 1.78 & 13.11 & 6.77 & -0.03 & 113.17 & 0.78 & -43.38 & 25.05 & -1.08 & $7.46 * *$ \\
\hline 54. & GAR-13 x IET-23834 & 79.00 & 2.27 & $15.46^{* *}$ & 8.02 & -0.93 & $2.99 * *$ & 102.34 & 2.96 & -34.87 & 20.46 & -0.57 & $5.16^{*}$ \\
\hline 55. & GNR-4 & 96.78 & 1.46 & $14.79 * *$ & 9.87 & 2.99 & -0.04 & 87.22 & -0.75 & 26.29 & 19.12 & 4.71 & -0.56 \\
\hline 56. & Kala Namak & 95.44 & -2.89 & $4.73^{*}$ & 7.96 & $2.30 *$ & -0.31 & 114.39 & 1.69 & 65.41 & 20.56 & 1.15 & $9.29 * *$ \\
\hline & Cross mean & 87.93 & & & 10.47 & & & 123.05 & & & 22.91 & & \\
\hline & General mean & 89.40 & 1.00 & & 9.92 & 1.00 & & 123.1 & 1.00 & & 21.87 & 1.00 & \\
\hline & S.E. \pm & 1.99 & 2.35 & & 0.79 & 2.48 & & 5.90 & 1.60 & & 1.14 & 3.88 & \\
\hline
\end{tabular}


Table.3 Contin...

\begin{tabular}{|c|c|c|c|c|c|c|c|c|c|c|c|c|c|}
\hline \multirow{2}{*}{$\begin{array}{l}\text { Sr. } \\
\text { No }\end{array}$} & \multirow[t]{2}{*}{ Genotypes } & \multicolumn{3}{|c|}{ Grains per panicle } & \multicolumn{3}{|c|}{ Grain yield per plant (g) } & \multicolumn{3}{|c|}{ Straw yield per plant (g) } & \multicolumn{3}{|c|}{ Test weight (g) } \\
\hline & & Mean & bi & $\mathbf{S}^{2} \mathrm{di}$ & Mean & bi & $S^{2} \mathbf{d i}$ & Mean & bi & $\mathrm{S}^{2} \mathrm{di}$ & Mean & bi & $\mathbf{S}^{2} \mathrm{di}$ \\
\hline 1. & NAUR-1 & 238.00 & 1.08 & 42.27 & 17.12 & 2.06 & $16.28^{*}$ & 20.54 & 2.07 & $23.39 * *$ & 23.68 & 9.90 & -1.17 \\
\hline 2. & GNR-3 & 224.26 & 6.64 & -37.36 & 18.80 & 3.25 & -2.24 & 22.57 & 3.16 & -1.59 & 30.52 & 4.04 & 0.59 \\
\hline 3. & Gurjari & 190.42 & 3.91 & $161.27 *$ & 12.59 & 1.95 & -2.95 & 15.11 & 1.90 & -2.98 & 26.66 & 0.53 & -0.51 \\
\hline 4. & GAR-13 & 223.00 & 2.57 & 56.03 & 12.78 & 1.33 & 2.87 & 15.34 & 1.34 & 4.90 & 20.24 & 0.84 & -0.95 \\
\hline 5. & NVSR-306-6 & 196.67 & 2.29 & -46.22 & 10.13 & 1.26 & -3.00 & 12.16 & 1.23 & -3.10 & 19.07 & 11.55 & 0.24 \\
\hline 6. & NVSR-306-14 & 207.68 & -0.69 & -24.13 & 10.24 & -0.60 & -0.55 & 12.29 & -0.61 & 0.30 & 21.52 & $-7.88^{*}$ & -1.31 \\
\hline 7. & IET-23814 & 187.58 & 1.33 & 122.83 & 11.66 & 0.79 & $12.02 *$ & 13.99 & 0.72 & $18.97 * *$ & 21.60 & -5.77 & -1.05 \\
\hline 9. & IET-23829 & 212.51 & -1.07 & $191.92 *$ & 10.85 & -0.53 & 2.31 & 13.02 & -0.49 & 4.71 & 21.36 & -5.32 & 0.00 \\
\hline 10. & IET-24767 & 255.91 & 0.41 & 77.32 & 23.92 & 1.83 & $35.17 * *$ & 28.70 & 1.70 & $53.43 * *$ & 21.83 & -1.19 & 0.58 \\
\hline 11. & IET-24774 & 205.12 & 3.02 & 1.65 & 14.92 & $2.39 * *$ & -2.99 & 17.90 & 2.34 & -3.12 & 20.62 & 9.47 & -0.54 \\
\hline 12. & IET-23832 & 225.83 & 3.11 & 5.36 & 13.32 & 1.11 & -2.99 & 15.98 & 1.08 & -3.09 & 18.77 & 9.55 & -0.69 \\
\hline 13. & IET-23833 & 262.20 & 1.23 & 60.95 & 22.14 & 0.68 & $23.54 * *$ & 26.57 & 0.59 & $35.49 * *$ & 23.55 & 3.96 & -0.37 \\
\hline 14. & IET-23834 & 210.53 & 1.32 & 67.76 & 15.07 & 1.86 & $13.96 *$ & 18.08 & 1.87 & $20.16^{* *}$ & 20.41 & $6.17 *$ & -1.31 \\
\hline & Parental mean & 217.59 & & & 14.55 & & & 17.46 & & & 22.31 & & \\
\hline 15. & NAUR-1 x NVSR-303-6 & 217.88 & 1.54 & $205.27 *$ & 19.30 & 1.30 & $9.56^{*}$ & 23.50 & 1.30 & 8.23 & 25.79 & $17.75^{*}$ & -1.28 \\
\hline 17. & NAUR-1 x IET-23814 & 235.18 & 1.33 & 114.16 & 20.26 & 0.78 & $10.71 *$ & 24.32 & 0.72 & $16.98^{*}$ & 24.72 & -2.33 & $4.91 *$ \\
\hline 18. & NAUR-1 x IET-23825 & 210.18 & $2.95^{*}$ & -48.19 & 17.74 & 2.48 & -2.89 & 21.29 & 2.42 & -3.06 & 25.50 & 8.61 & -0.12 \\
\hline 19. & NAUR-1 x IET-23829 & 242.33 & 2.24 & -10.38 & 19.26 & 2.88 & -2.90 & 23.11 & 2.81 & -2.83 & 21.72 & -14.22 & -0.26 \\
\hline 20. & NAUR-1 x IET-24767 & 265.53 & 1.62 & $156.03^{*}$ & 28.53 & 1.64 & $20.45 * *$ & 34.24 & 1.54 & $31.65 * *$ & 27.83 & -7.14 & 2.02 \\
\hline 21. & NAUR-1 x IET- 24774 & 246.76 & -0.20 & -15.25 & 24.08 & -0.73 & 8.20 & 27.23 & 1.19 & 5.94 & 23.89 & 11.16 & -1.20 \\
\hline 22. & NAUR-1 x IET-23832 & 225.67 & -2.75 & -7.19 & 21.86 & $-1.58^{*}$ & -3.00 & 26.23 & $-1.54 *$ & -3.12 & 25.46 & -0.17 & -0.23 \\
\hline 23. & NAUR-1 x IET-23833 & 271.05 & 4.69 & $207.81 *$ & 27.75 & 2.60 & -1.82 & 33.29 & 2.53 & -1.03 & 26.71 & -8.34 & 2.94 \\
\hline 24. & NAUR-1 x IET-23834 & 227.90 & 2.27 & -43.78 & 23.02 & 1.95 & -2.81 & 27.62 & 1.89 & -2.72 & 22.74 & -2.08 & $4.68 *$ \\
\hline 25. & GNR-3 x NVSR-303-6 & 229.33 & -0.91 & -27.44 & 22.68 & -0.58 & $15.21 *$ & 28.55 & 0.67 & $9.93 *$ & 24.68 & -9.61 & -0.76 \\
\hline 26. & GNR-3 x NVSR-303-14 & 222.24 & -0.16 & 53.24 & 21.72 & -0.63 & $8.75^{*}$ & 26.06 & -0.57 & $14.04 *$ & 22.40 & 0.12 & $7.60 * *$ \\
\hline 27. & GNR-3 x IET-23814 & 238.00 & 1.52 & -0.70 & 22.56 & 2.24 & -2.45 & 27.41 & 1.88 & -2.79 & 25.04 & $14.75^{*}$ & -1.31 \\
\hline 28. & GNR-3 x IET-23825 & 215.95 & 2.25 & -39.22 & 21.37 & 2.49 & -2.78 & 25.65 & 2.43 & -2.63 & 24.48 & 7.92 & 0.22 \\
\hline
\end{tabular}




\begin{tabular}{|c|c|c|c|c|c|c|c|c|c|c|c|c|c|}
\hline \multirow{2}{*}{$\begin{array}{l}\text { Sr. } \\
\text { No }\end{array}$} & \multirow[t]{2}{*}{ Genotypes } & \multicolumn{3}{|c|}{ Grains per panicle } & \multicolumn{3}{|c|}{ Grain yield per plant (g) } & \multicolumn{3}{|c|}{ Straw yield per plant (g) } & \multicolumn{3}{|c|}{ Test weight (g) } \\
\hline & & Mean & bi & $\mathbf{S}^{2} \mathbf{d i}$ & Mean & bi & $\mathbf{S}^{2} \mathbf{d i}$ & Mean & bi & $S^{2} \mathrm{di}$ & Mean & bi & $\mathbf{S}^{2} \mathbf{d i}$ \\
\hline 29. & GNR-3 x IET-23829 & 234.31 & 1.46 & -16.95 & 18.73 & 1.23 & 1.41 & 22.48 & 1.23 & 2.92 & 21.46 & 10.04 & -0.65 \\
\hline 30. & GNR-3 x IET-24767 & 276.05 & 2.56 & $258.51^{*}$ & 30.40 & 1.57 & $11.07 *$ & 36.18 & 1.85 & $12.99^{*}$ & 26.13 & 14.91 & -0.32 \\
\hline 31. & GNR-3 x IET-24774 & 225.81 & 2.62 & -16.43 & 25.18 & 3.04 & -2.10 & 30.88 & 2.35 & -2.95 & 22.07 & 7.97 & 1.72 \\
\hline 32. & GNR-3 x IET-23832 & 203.41 & 1.25 & -18.47 & 23.49 & 2.09 & -2.95 & 28.19 & 2.04 & -2.97 & 25.35 & -3.92 & 2.59 \\
\hline 33. & GNR-3 x IET-23833 & 223.12 & 2.34 & -44.55 & 26.26 & 2.41 & -2.79 & 31.51 & 2.35 & -2.65 & 26.55 & 11.21 & 0.60 \\
\hline 34. & GNR-3 x IET-23834 & 225.21 & -1.96 & 95.38 & 17.81 & $-1.95^{*}$ & -2.98 & 21.37 & $-1.90 *$ & -3.04 & 24.10 & -15.76 & -0.94 \\
\hline 35. & Gurjari x NVSR-303-6 & 189.44 & -1.68 & -10.33 & 15.81 & -0.65 & 5.91 & 18.97 & -0.68 & $9.40 *$ & 19.23 & $-17.31 *$ & -1.27 \\
\hline 37. & Gurjari x IET-23814 & 208.63 & -0.36 & 33.49 & 17.79 & -0.62 & 6.17 & 21.34 & -0.56 & $10.33^{*}$ & 20.14 & 9.03 & 3.03 \\
\hline 38. & Gurjari x IET-23825 & 183.20 & -2.56 & 40.13 & 15.43 & -0.55 & 3.67 & 18.51 & -0.57 & 6.28 & 18.24 & -11.60 & 0.85 \\
\hline 39. & Gurjari x IET-23829 & 209.76 & -0.58 & 6.14 & 15.25 & -0.24 & 1.41 & 18.32 & -0.20 & 3.12 & 16.63 & -5.93 & 0.98 \\
\hline 40. & Gurjari x IET-24767 & 248.56 & 1.95 & -46.66 & 25.82 & 2.34 & -2.62 & 30.98 & 2.28 & -2.37 & 24.77 & -10.75 & -0.91 \\
\hline 41. & Gurjari x IET-24774 & 200.87 & -1.27 & 6.43 & 18.83 & 2.25 & -2.61 & 22.93 & 1.88 & -2.94 & 20.82 & 14.58 & -0.85 \\
\hline 42. & Gurjari x IET-23832 & 186.92 & -1.63 & -45.94 & 16.61 & $-1.17 *$ & -2.99 & 19.93 & $-1.14^{*}$ & -3.07 & 18.57 & 3.51 & 1.17 \\
\hline 43. & Gurjari x IET-23833 & 249.49 & -0.67 & 71.93 & 25.96 & $2.53^{*}$ & -2.97 & 31.48 & $2.17 *$ & -3.12 & 25.77 & 7.45 & -1.29 \\
\hline 44. & Gurjari x IET-23834 & 153.51 & 2.43 & -12.00 & 17.77 & 2.07 & -2.96 & 21.33 & 2.02 & -2.99 & 18.19 & 6.96 & 2.46 \\
\hline 47. & GAR-13 x IET-23814 & 174.52 & -2.24 & 70.83 & 15.79 & -0.80 & 3.19 & 18.95 & -0.81 & 5.52 & 15.68 & -4.52 & -0.67 \\
\hline 48. & GAR-13 x IET-23825 & 150.85 & 3.51 & -35.23 & 15.25 & 1.68 & -2.83 & 18.29 & 1.64 & -2.76 & 17.67 & -7.62 & -1.27 \\
\hline 49. & GAR-13 x IET-23829 & 184.39 & -2.02 & -43.66 & 16.09 & -1.16 & -2.09 & 19.31 & -1.12 & -1.67 & 21.57 & -10.01 & -0.58 \\
\hline 50. & GAR-13 x IET-24767 & 245.83 & 1.36 & -1.96 & 28.09 & 1.34 & 7.92 & 33.71 & 1.36 & $12.01 *$ & 24.59 & 7.56 & 0.57 \\
\hline 51. & GAR-13 x IET-24774 & 170.27 & 1.15 & 55.75 & 17.94 & -1.38 & -2.39 & 23.86 & -0.33 & $11.00^{*}$ & 19.54 & -9.90 & -0.58 \\
\hline 52. & GAR-13 x IET-23832 & 159.74 & 1.83 & 12.87 & 15.14 & 1.58 & -2.58 & 18.17 & 1.54 & -2.37 & 15.75 & 0.24 & -0.19 \\
\hline 53. & GAR-13 x IET-23833 & 242.26 & $2.83^{*}$ & -47.80 & 26.62 & $2.83^{*}$ & -3.00 & 31.95 & $2.76^{*}$ & -3.10 & 25.53 & 8.98 & -1.14 \\
\hline 54. & GAR-13 x IET-23834 & 175.21 & $-2.77 *$ & -47.84 & 16.08 & -0.54 & $9.31 *$ & 19.30 & -0.48 & $14.84^{*}$ & 16.79 & -6.88 & -1.25 \\
\hline 55. & GNR-4 & 223.76 & 2.99 & -7.94 & 20.54 & 1.32 & -2.75 & 22.35 & 0.02 & 1.33 & 19.06 & 1.17 & -1.21 \\
\hline 56. & Kala Namak & 199.92 & 2.72 & 13.63 & 15.35 & $2.35 * *$ & -3.00 & 18.43 & $2.29 *$ & -3.12 & 22.69 & 3.06 & -0.68 \\
\hline & Cross mean & 213.19 & & & 20.40 & & & 24.59 & & & 22.00 & & \\
\hline & General mean & 214.20 & 1.00 & & 18.85 & 1.00 & & 22.66 & 1.00 & & 22.03 & 1.00 & \\
\hline & S.E. \pm & 6.30 & 1.80 & & 1.71 & 1.32 & & 2.02 & 1.27 & & 0.90 & 8.57 & \\
\hline
\end{tabular}


Int.J.Curr.Microbiol.App.Sci (2017) 6(11): 2102-2111

Table.4 Stable hybrids identified on the basis of high means for grain yield per plant and other characters over three and specific environments

\begin{tabular}{|c|c|c|c|c|c|}
\hline $\begin{array}{l}\text { Sr. } \\
\text { No. }\end{array}$ & Hybrids & $\begin{array}{l}\text { Grain yield } \\
\text { per plant }(g)\end{array}$ & $\begin{array}{l}\text { bi } \\
\text { value }\end{array}$ & Nature of adaptation & Average stable \\
\hline 1 & $\begin{array}{l}\text { GAR-13 } \quad \mathrm{x} \text { IET- } \\
24767\end{array}$ & 28.09 & 1.34 & $\begin{array}{l}\text { Average responsive suitable } \\
\text { for environment and } \\
\text { recommended }\end{array}$ & PH, PL, GPP, GY, TW \\
\hline 2 & $\begin{array}{lll}\text { NAUR-1 } & x \quad \text { IET- } \\
23833 & & \end{array}$ & 27.75 & 2.60 & $\begin{array}{l}\text { Average responsive suitable } \\
\text { for environments }\end{array}$ & $\begin{array}{l}\text { DF, PPP, PH, PL, GY, } \\
\text { SY, TW }\end{array}$ \\
\hline 3 & $\begin{array}{l}\text { GAR-13 } x \text { IET- } \\
23833\end{array}$ & 26.62 & $2.83^{*}$ & $\begin{array}{l}\text { Highly responsive suitable } \\
\text { for favourable environments }\end{array}$ & DF, PPP, PH, TW \\
\hline 4 & GNR-3 x IET-23833 & 26.26 & 2.41 & $\begin{array}{l}\text { Average responsive suitable } \\
\text { for environments }\end{array}$ & $\begin{array}{l}\text { DF, PPP, PH, PL, GPP, } \\
\text { GY, SY, TW }\end{array}$ \\
\hline 5 & Gurjari x IET-23833 & 25.96 & $2.53^{*}$ & $\begin{array}{l}\text { Highly responsive suitable } \\
\text { for favourable environments }\end{array}$ & PPP, PH, GPP, TW \\
\hline
\end{tabular}

$\mathrm{DF}=$ Days to $50 \%$ flowering

$\mathrm{GPP}=$ Grains per panicle
PPP = Productive tillers per plant

$\mathrm{GY}=$ Grain yield per plant $(\mathrm{g})$
$\mathrm{PH}=$ Plant height $(\mathrm{cm})$

$\mathrm{SY}=$ Straw yield per plant $(\mathrm{g})$
$\mathrm{PL} \quad=\quad$ Panicle length $(\mathrm{cm})$

$\mathrm{TW} \quad=$ Test weight $(\mathrm{g})$ 
Stability was observed in some of the genotypes by Belhekar (2012) and Subudhi et al., (2012). Taking these parameters into consideration, the results obtained are discussed character wise.

\section{Days to 50 per cent flowering}

Among parents lowest mean value (81.11) accompanied by non-significant bi (2.49) as well as least deviation from zero (0.70) was found for IET-23833, identified as a stable line. Among 40 rice hybrids, NAUR-1 x IET23833 showed early flowering with nonsignificant bi value and minimum deviation from regression classified as highly stable hybrid across all the environments followed by GNR-3 x IET-23833 and NAUR-1 x IET23834 (Table 3).

\section{Number of productive tillers per plant}

Four parental line viz., NAUR-1, IET-24767, IET-24774 and IET-23833 exhibited higher mean value, bi value near unity and nonsignificant $S^{2}$ di, shows average stability. Out of 40 hybrids, ten hybrids were constituted to be stable for this trait. Among these stable hybrids, best three were NAUR-1 $x$ IET23833, GNR-3 x IET-23833 and GNR-3 x IET-24767. GNR-3 x IET-23832 hybrid found to be more stable in rich environment (Table 3).

\section{Plant height (cm)}

For plant height, mean sum of square due to $\mathrm{G} \times \mathrm{E}$ interaction was observed to be nonsignificant (Table 1). It revealed that all parents and hybrids along with standard checks were consistently performing across environments (Table 3).

\section{Panicle length $(\mathrm{cm})$}

Among the parents, NAUR-1, GNR-3, GAR13, IET-24767 and IET-23833 showed higher mean value than parental mean and displayed average stability over environment for this character (bi>1 and S2di: 0). Total 19 hybrids manifested higher mean value, bi around unity and non-significant $S^{2}$ di identified as stable across the environments. The best three stable hybrids were NAUR-1 x IET-23833, GNR-3 x IET-23833 and NAUR-1 x IET24767. None of the hybrids showed specific adaptability for this trait (Table 3 ).

\section{Numbers of grains per panicle}

Three lines viz., NAUR-1, GNR-3 and GAR13 and three testers viz., IET-24767, IET23832 and IET-23833 mark highest number of grains per panicle, with unit bi value and non-significant S2di, hence were considered average stable over environments. Hybrid GAR-13 x IET-23833 identified as stable in rich environment with significant regression greater than unity. In contrast, GAR-13 x IET-23834 had significant regression less than one, classified as low responsive and better in unfavourable environment. The best three hybrids based on their stable performance over environments were Gurjari $x$ IET-23833, Gurjari $x$ IET-24767 and NAUR-1 x IET-24774 (mean > general mean, bi: 1 and $S^{2}$ di: 0) (Table 3).

\section{Grain yield per plant (g)}

Line GNR-3 exhibited greater mean than general men along with regression coefficient near unity and non-significant $S^{2}$ di, classified as average responsive suitable for all environment and tester IET-24774 suitable for favourable environment (Table 4).

Highest yield and average stable hybrids were observed for GAR-13 x IET-24767 followed by NAUR- 1 x IET-23833 and GNR-3 x IET23833. The hybrid NAUR-1 x IET-23832 showed above average stability best in poor environment. Likewise the hybrids Gurjari $\mathrm{x}$ IET-23833 and GAR-13 x IET-23833 displayed specific adaptability toward 
favourable environment. Grafius (1959) also reported similarly (Table 3 ).

\section{Straw yield per plant (g)}

Among the parents, GNR-3 and IET-24774 exhibited stability across the environments. Whereas among the hybrids, nine hybrids exhibited average stability across the environments. Two hybrids, Gurjari x IET23833 and GAR-13 x IET-23833 showed high mean value, bi value significantly greater than unity and non-significant $S^{2}$ di suggesting that these hybrids were stable for favourable environments. On the other hand, hybrid NAUR-1 x IET-23832 depicted high mean value, bi value significantly lower than unity and non-significant $S^{2}$ di indicating that these were stable for poor environments (Table 3 ).

\section{Test weight (g)}

The mean sum of square due to $G \times \mathrm{E}$ interaction was recorded to be non-significant for this trait. This revealed consistent performance of this trait across the environments for all forty genotypes (Table $3)$. The most stable five hybrids are listed in Table 4 along with their grain yield per plant and component trait for which they had exhibited stability. The best five stable hybrids viz., GAR-13 x IET-24767, NAUR-1 x IET-23833, GAR-13 x IET-23833, GNR-3 $x$ IET-23833 and Gurjari x IET-23833 had higher grain yield per plant and higher stability for some characters.

In conclusion, the present study provided an evaluation of genotypic and environmental performance of forty hybrids of rice over varied environments. Stability analysis demonstrated that hybrids viz., GAR-13 $\mathrm{x}$ IET-24767, NAUR-1 x IET-23833 and GNR$3 \times$ IET-23833 is average responsive suitable for all environment while two hybrids, GAR$13 \times$ IET-2383 and Gurjari x IET-23833 is highly responsive suitable for favourable environment in terms of yield.

\section{References}

Belhekar, P. B. (2012). Genetic analysis in aromatic rice (Oryza sativa L.) with cytoplasmic genetic male sterile lines. Ph.D. thesis submitted to Navsari Agricultural University, Navsari.

Eberhart, S.A. and Russell, W.A. (1966). Stability parameters for comparing varieties. Crop Sci., 6(1): 36-40.

Grafius, J. E. (1959). Heterosis in barley. Agron. J., 51: 515-554.

Guyer, D., Tuttle, A., Rouse, S., Volrath, S., Johnson, M., Potter, S., Gorlach, J., Goff, S., Crossland, L., and Ward, E. (1998). Activation of latent transgenes in arabidopsis using a hybrid transcription factor. Genet., 149: 633-639.

Nayak, A.R. (2008). Stability of quality characters in scented rice. Ind. J. Agric. Res., 24(2): 102-106.

Shadakshari, Y.G., Chandrappa, H.M., Kulkarni, R.S. and Shashidher, H.E. (2001). Genotypic X Environment interaction in low land rice genotypes of hill zone of Karnataka. Ind. J. Genet., 64(4): 350-352.

Subudhi, H. N., Bose, L. K., Singh, O.N. and Rao, G.J. N. (2012). Genotype x Environment interaction for grain yield and its component traits in irrigated rice. Madras Agric. J., 99(4-6): 178-180.

\section{How to cite this article:}

Patel Unnati, Patel Pathik, Mahatma Lalit and Bhimshinh Bilwal. 2017. Stability Analysis for Grain Yield and Its Attributing Traits of Rice across Locations. Int.J.Curr.Microbiol.App.Sci. 6(11): 2102-2111. doi: https://doi.org/10.20546/ijcmas.2017.611.248 